hielt Vorträge, veröffentlichte regelmäßig in Tageszeitungen zu Leserfragen. Bis 1990 war sie es, die als Notarin beim Fernsehen der DDR bei der Ziehung von Lottozahlen und im Telelotto Beurkundungen vornahm. In 33 Fernsehunterhaltungssendungen von „Wennschon-Dennschon“ war sie als Juryvorsitzende tätig. ${ }^{42}$ Alle in der DDR kannten sie.

Zunehmend wurden GmbHs und AGs gebraucht, weil unter dem Namen „Volkseigentum“ jede volkseigene Fabrik für jede andere gepfändet werden konnte. In dem „weißen Haus am Bahnhof Friedrichstraße (da saßen sie alle, z. B. Schalck-Golodkowski mit seiner Kunst- und Antiquitäten$\mathrm{GmbH}$ )“ musste beurkundet werden. Ihr fiel sozusagen spiegelverkehrt die Rolle zu, die am Anfang der DDR Ingeburg Gentz innehatte.

"Das Ministerium kam zu mir. Ich hatte nie eine GmbH gesehen oder begründet. Nur ein alter Rechtspfleger in Berlin konnte das noch. Das Ministerium konnte einem noch nicht einmal die Formularbücher aus dem Westen besorgen. "

So ging sie zu dem Rechtspfleger, kündigte eine Revision an, nahm die Verträge zur GmbH-Gründung mit und kopierte sie. Ergänzt durch etwas Literatur aus der Humboldt-Universität wurde das die Grundlage für „Beurkundungen im Handelshaus“. Dann kam die Wende, und ihre gerade erworbenen Kenntnisse trugen ungeahnte Früchte. Ganze VEBs mussten bis zur Währungsunion am 1. Juli 1990 in GmbHs umgewandelt werden. An zwei Wochenende bewältigte sie das Schicksal der gesamten Schuhindustrie der DDR.

\section{Rückblick}

Die winzige Berufsgruppe der Notar/inn/e/n - zum Zeitpunkt der Vereinigung waren es insgesamt 379, davon 257 Frau- $e^{43}$ - wird nie im Rampenlicht der DDR-Justizgeschichte bzw. deren Justizgeschichtsschreibung stehen. Es bedurfte des sorgfältigen Nachforschens vermittels verstreuter ArchivDokumente, vor allem aber persönlicher Gespräche mit den damals selbst Beteiligten, um über bloße statistische und andere offizielle Angaben hinaus dem Wesen dieses Notariats nachzuspüren. Die Frauen, die sich zu solchen Gesprächen bereiterklärten (sowohl solche, die nach 1990 den Schritt in die Selbstständigkeit gewählt hatten, als auch andere, ältere, deren Berufsleben mit dem Ende der DDR zusammenfiel) berichteten keineswegs unkritisch, aber mit Engagement und Wärme von ihrer Tätigkeit. Gemeinsamer Nenner waren die (relative) Selbstständigkeit bei der Arbeit sowie das Gefühl, dass diese Arbeit trotz politisch vorgegebenem engen Rahmen den Rat suchenden Bürgerinnen und Bürgern „etwas brachte“. Arbeits- und Lernwille sowie fürsorgliches Interesse an den Sorgen der Mitbürger, nicht aber herausragende juristische Fähigkeiten waren bei der Ausübung ihres Berufs gefragt. Desto stärker beeindrucken Persönlichkeit und Leistung der hier je einzeln vorgestellten Notarinnen Dr. Ingeburg Gentz und Sabine Herrmann.

42 Herrmann, Sabine, in Huebner, Ralph (Hrsg.), Who Is Who in der Bundesrepublik Deutschland. Supplementwerk der biographischen Enzyklopädie führender Frauen und Männer Deutschlands, Verlag für Personenenzyklopädien 1998.

43 Bundesnotarkammer, Notarverzeichnis der Bundesländer Ost 1990.

\title{
Rechtsanwältinnen in der DDR
}

\section{Dr. Stefanie Loroch}

Münster

Entgegen manch düsterer Prophezeiung ${ }^{1}$ entpuppte sich die Zulassung der Frauen zur Anwaltschaft vor 90 Jahren keineswegs als Schaden, vielmehr als Segen für die Rechtspflege. Justizrat Dr. Bieber, der 1922 anlässlich der 14. Vertreterversammlung der Anwaltschaft die vermeintliche Nichteignung der Frau zum Juristenberuf schlicht als Vorurteil abtat, behielt Recht. ${ }^{2}$ Die Geschichte hat die Gegenargumente von einst überholt: Justitia ist eine Frau und der Rechtsanwalt immer häufiger weiblich.

Fast ein Drittel der zugelassenen Rechtsanwältinnen und Rechtsanwälte sind nach der im Jahr 2011 veröffentlichten Statistik der Bundesrechtsanwaltskammer Frauen, Tendenz steigend. ${ }^{3}$ Dies ist ein nicht zu unterschätzender Wert, denn der Zeitraum seit Zulassung der ersten Rechtsanwältin im Jahr 1922 ist überschaubar kurz.
Der Nationalsozialismus beendete vorläufig, was gerade erst begonnen hatte. Nach dem Ende des zweiten Weltkrieges begann eine Phase des Wiederaufbaus und der politischen Umstrukturierung. Die mit der Einteilung in die Besatzungszonen einhergehende Rechtszersplitterung bedingte letztlich ein Auseinanderdriften der Anwaltschaft im Ostteil und im Westteil Deutschlands. Damit nahm auch die Geschichte der Rechtsanwältinnen in der BRD und der DDR einen unterschiedlichen Verlauf.

\section{Der weibliche Rechtsanwalt in der DDR}

„Sie geht davon aus, daß sie als ausgebildeter Jurist einen Anspruch darauf hat, ihren persönlichen Wünschen entsprechend

\footnotetext{
1 Vgl. Protokoll der 14. Vertreterversammlung der Anwaltschaft 1922, in: JW 1922, S. 1246 ff.

2 Protokoll der 14. Vertreterversammlung der Anwaltschaft 1922, in: JW 1922, 1246 ff. (S. 1247-1249).

3 Stand 1.1.2011: 32,04\%.
} 
eingesetzt zu werden. “4 - ein Trugschluss, wie einem Antwortschreiben des Ministeriums der Justiz aus dem Jahr 1983 auf eine Anfrage der Volkskammer zu entnehmen war. Die Antragstellerin hatte im Fernstudium Jura studiert, betätigte sich nach Informationen des Ministeriums bereits beratend und begehrte mehrfach mit Nachdruck und unter Anrufung verschiedener Stellen die Zulassung als Rechtsanwältin - ohne Erfolg. Die juristische Qualifikation allein genügte nicht, um die Aufnahme zur Rechtsanwaltschaft in der DDR beanspruchen zu können.

Nachdem auf der 2. Parteikonferenz der SED im Juli 1952 offiziell der Aufbau des Sozialismus deklariert worden war, vollzog sich in der DDR eine umfassende Verwaltungs- und Justizreform. ${ }^{5}$ Propagiert wurde das Leitbild der sowjetischen Anwaltschaft. Eine Anwaltschaft, die sich als „Instrument im Kampf für den Sozialismus“ begreife, ${ }^{6}$ nicht immer neutral, sondern politisch-ideologisch kontrolliert und diszipliniert, wie Fricke feststellt. ${ }^{7}$

Durch eine Verordnung vom 15. Mai 1953 wurde die Bildung von Kollegien der Rechtsanwälte beschlossen und damit, so Fricke, ein „radikaler Wandel“ bewirkt. ${ }^{8}$ Das Ziel war, wie es in der Präambel hieß, den Anwälten in der DDR zu ermöglichen, „in der unserer Gesellschaftsordnung entsprechenden Form bei der Festigung der volksdemokratischen Grundlagen unseres Staates mitzuwirken “.9 Mit dem der Verordnung beigefügten Musterstatut sollte „den intensiven Bestrebungen der fortschrittlichen Rechtsanwälte und ihrem Verlangen nach kollektiven Zusammenschlüssen“ die gesetzliche Grundlage gegeben werden. Entgegen dieser Verlautbarung war das Verlangen der Anwälte in dieser Deutlichkeit jedoch keineswegs zu erkennen. Lorenz äußert vielmehr die Ansicht, dass nicht die Anwälte selbst, sondern eine gezielte Hintergrundarbeit des Ministeriums der Justiz in Absprache mit den jeweiligen SED-Bezirksleitungen die Richtung vorgab. ${ }^{10}$ Er beruft sich unter anderem auf einen Bericht der damals neu ernannten Ministerin der Justiz Hilde Benjamin vom 3. August 1953, in dem es hieß: „Es ist festzustellen, daß zur Zeit nur eine geringe Neigung zur Kollegiumsbildung besteht." 11 Und so waren Ende 1953 von insgesamt 840 Rechtsanwälten nur etwa 17 Prozent Mitglieder eines Kollegiums. ${ }^{12}$ Die Zahl der frei praktizierenden Anwälten sollte im Laufe der folgenden Jahre jedoch deutlich abnehmen.

Die Kontrollmechanismen des Kollektivs waren durch die Revisionskommissionen und parteiliche Einflussnahme eng geschnürt. Die Anleitung und Aufsicht über die Tätigkeit der Kollegien und Einzelanwälte oblag offiziell dem Ministerium der Justiz. Das Ministerium entschied über Zulassung und Einsatzort. Im Rahmen der sogenannten „Absolventenlenkung“ wurden die frisch gebackenen Uni-Absolvent/inn/en in den Kollegien untergebracht, die zuvor Bedarf angemeldet hatten.

Trotz Lenkung von ministerieller Seite lief die praktische Einrichtung der Kollegien in der Anfangsphase nicht immer unproblematisch ab. In Karl-Marx-Stadt beispielsweise bereitete die Raumbeschaffung besondere Schwierigkeiten. Diese konnten erst durch „viele Verhandlungen überbrückt“ werden, monierte das Kollegium im Nachhinein. Erst ab November 1954 standen auf der Dresdner Straße im Hinterhaus vier Räume zur Verfügung, nur zwei waren beheizbar und mit Fenstern versehen. „Mehr als primitiv“ - lautete das geringschätzige Urteil des Kollegiums. ${ }^{13}$

\section{Zielbewusste Aufnahmepolitik}

Ob männlich oder weiblich - gemäß der provisorischen $\mathrm{Zu}$ lassungsordnung musste ein/e Bewerber/in die für Richter/ innen vorgeschriebenen Anforderungen des $\$ 2$ GVG vom 27. Januar $1877^{14}$ erfüllen. Trotz gleicher Bedingungen blieben Frauen in der Anwaltschaft vorerst die Ausnahme.

Doch die Berufstätigkeit der Frau wurde vom SED-Regime propagiert, die Emanzipation staatlich forciert. Der Ministerrat forderte 1963 die Leiter der Staats- und Wirtschaftsorgane auf, den Einsatz und die Leistungen der Frauen ihres Verantwortungsbereichs zu analysieren und entsprechende Maßnahmen einzuleiten. Knapp zwei Jahre später ist in einem Schriftsatzentwurf an den Ministerrat zu lesen: „Nach wie vor wird im Rahmen der Kaderverantwortlichkeit des Ministeriums der Justiz der Förderung und Entwicklung der Frau in der Justiz größte Bedeutung beigemessen und alles getan, die Tätigkeit der Frau bei den Gerichten und Staatlichen Notariate sowie im Ministerium der Justiz allseitig zu unterstützen. " ${ }^{15}$ In einer Hausmitteilung des Ministeriums der Justiz aus dem gleichen Jahr wurde ferner die Notwendigkeit festgehalten, auch bei der Auswahl der Rechtspflegestudenten darauf zu achten, den Anteil der Frauen weiter zu erhöhen. ${ }^{16}$

Das Ministerium der Justiz hatte zudem ein Programm für die Auswertung der Erhebung unter den weiblichen Juristinnen in der Justiz erstellt. Der hierzu entworfene Fragebogen umfasste Aspekte wie Alter, Gesundheitszustand, Ausbildung, aber auch Zufriedenheit, Wunsch nach höherer Funktion, Zeit für Weiterbildung, Forderung nach Teilbeschäftigung, Belastung durch Haushalt und Familie und Hilfe durch Ehemänner.

4 Schreiben an die Volkskammer v. 1.7.1983, Bundesarchiv: DP-1/416o.

5 Brand, Peter-Andreas, Der Rechtsanwalt und der Anwaltsnotar in der DDR, Köln 1985, S. 31

6 Kohn, Karl, Die Rechtsanwaltschaft in der Sowjetunion, in: NJ 1950, 192 (S. 193).

7 Fricke, Karl Wilhelm, Praxis der Anwaltstätigkeit in der SBZ und in der DDR, in: DAV (Hrsg.), Anwälte und ihre Geschichte, Tübingen 2011, S. 475 f.

8 Fricke, Karl Wilhelm, Praxis der Anwaltstätigkeit in der SBZ und in der DDR, in: DAV (Hrsg.), Anwälte und ihre Geschichte, Tübingen 2011, S. 473.

9 Gesetzblatt der DDR II, 1953, S. 725.

10 Lorenz, Thomas, Die Rechtsanwaltschaft in der DDR, Berlin 1998, S. 127.

11 Ebd. S. 128 mit Verweis auf das Bundesarchiv: DP-1/1016/322-323.

12 Ders., Die „Kollektivierung“ der Rechtsanwaltschaft, in: Rottleuthner, Hubert, Steuerung der Justiz in der DDR, Köln 1994, S. 409-428 (S. 417).

13 Quartalsübersicht des Kollegiums der Rechtsanwälte Karl-MarxStadt v. 5.11.1954, Bundesarchiv: DP-1/480.

14 RGBI. $1877,41 \mathrm{ff}$.

15 Schriftsatzentwurf an den Ministerrat der DDR zum Beschluss des Ministerrats v. 21.1.1965 über den Bericht über die Verwirklichung der Vorschläge und Empfehlungen des Frauenkongresses der DDR v. 16.2.1965, Bundesarchiv: DP-1/1653.

16 Hausmitteilung des Ministeriums der Justiz v. 3.2.1965, Bundesarchiv: DP-1/1653. 
Es sollte zudem untersucht werden, ob Beziehungen zwischen der sozialen Belastung der weiblichen Juristinnen und dem beruflichen Streben bestehen. ${ }^{17}$

Der hohe Anteil der Frauen in der Justiz - etwa zwei Drittel - wird in einem Bericht aus dem Jahr $1968^{18}$ als „erfreulich “ bezeichnet, die Ausgangsposition für Frauen insbesondere im Hinblick auf leitende Funktionen sei jedoch noch verbesserungsbedürftig, so das Fazit. ${ }^{19}$

Dieses prozentuale Kräfteverhältnis der Geschlechter spiegelte sich in der Anwaltschaft allerdings nicht wider, hier war der Frauenanteil weit geringer: „Der Anteil der weiblichen Rechtsanwälte im Bestand der Mitgliedschaft des Kollegiums ist angemessen zu erhöhen. “20 - lautete demgemäß die Vorgabe des Kaderprogramms des Anwaltskollegiums Leipzig und zwar noch im Jahr 1981.

Wann die erste Rechtsanwältin in der DDR zugelassen wurde, lässt sich anhand der gesichteten Unterlagen nicht eindeutig bestimmen. ${ }^{21}$ Das Kollegium in Dresden beispielsweise stellte in seinem Bericht über die Tätigkeit des Vorstandes im 1. Halbjahr 1964 fest, von den insgesamt 46 tätigen Rechtsanwält/inn/en seien fünf weiblich. ${ }^{22}$ In den Aufzeichnungen der Rechtsanwaltskammer Sachsen findet sich für das damalige Gebiet des heutigen Kammerbezirks die erste Rechtsanwältin im Jahr 1963, 1964 kam eine weitere, 1971 kamen zwei weitere Kolleginnen hinzu. ${ }^{23}$

Nach einer Entwurfvorlage für die Kollegiumssitzung am 12. Mai 1965 über die Lage der Rechtsanwaltschaft in der DDR waren 195836 und 196437 Anwältinnen tätig. Während sich die Zahl in den Kollegien von 24 auf 32 erhöht habe, sei sie bei den Einzelanwälten von zwölf auf fünf und bei den Rechtsbeiständen von acht auf vier zurückgegangen. ${ }^{24}$

„Auffällig ist, daß von keinem einzigen Kollegium eine Frau für den Vorstand nominiert und in den Vorstand gewählt worden ist", wurde im Protokoll der Jahreshauptversammlung der Rechtsanwälte 1964/65 vermerkt. ${ }^{25}$ Mit Ausnahme der Kollegien Erfurt, Potsdam und Rostock, die je eine Frau in die Revisionskommission gewählt hatten, traf das auch auf die Zusammensetzung der Revisionskommissionen der übrigen Rechtsanwaltskollegien zu. Hierzu hält das Sitzungsprotokoll fest: „Diese Tatsache scheint den Mitgliedern der betreffenden Kollegien bisher kaum bewußt geworden zu sein.“ Lediglich zwei Kolleginnen aus dem Kollegium Berlin hätten zu diesem wichtigen Punkt Stellung genommen. ${ }^{26}$ Als Aufgabe aller Kollegien wurde angesichts dessen gefordert, eine „zielbewußte Aufnahmepolitik“ zu betreiben, „insbesondere mit dem Ziel, eine Verjüngung der Rechtsanwaltschaft herbeizuführen, wobei gleichzeitig eine Vergrößerung des Frauenanteils anzustreben ist. “27

Dieses Streben führte in den Folgejahren zu einer mancherorts sichtbaren Erhöhung des Frauenanteils in der Anwaltschaft: In Potsdam beispielsweise waren 1976 insgesamt 34 Rechtsanwältinnen und -anwälte zugelassen. Der Frauenanteil betrug immerhin 9 Prozent. ${ }^{28}$ Spitzenreiter war 1983 das Kollegium Cottbus, das zu diesem Zeitpunkt mit 25 Prozent den höchsten Frauenanteil hatte. ${ }^{29}$
Zwei Jahre zuvor, 1981, hatte das Kollegium Leipzig in seinem Kaderprogramm den Frauenanteil untersucht und zahlenmäßig aufgeschlüsselt. Danach waren immerhin zehn der 49 Anwält/inn/e/n Frauen. Dennoch war auch weiterhin erklärtes Ziel, insbesondere den Anteil der Frauen in Leitungsfunktionen zu erhöhen. Die Vorschlagsliste für die Besetzung der Posten in Parteileitung, Vorstand, Revisionskommission und Rechtsausschuss enthielt neben den 22 Männer- zumindest auch fünf Frauennamen. ${ }^{30}$

Angesichts dieser Zahlen ist daher zusammenfassend festzustellen, dass der prozentuale Anteil der Anwältinnen von Kollegium zu Kollegium unterschiedlich war. Wurde in Cottbus und Leipzig bereits zu Beginn der Achtzigerjahre die 20-Prozent-Grenze überschritten, wies der Kaderperspektivplan des Kollegiums Schwerin zu Beginn des Jahres 1987 nur 13,64 Prozent Anwältinnen aus, und auch die Vorstandsämter waren noch sämtlich in männlicher Hand. ${ }^{31}$ Das Kollegium Magdeburg ließ sogar erst 1983 die erste Anwältin zu.

Während die Kollegien in Karl-Marx-Stadt und Leipzig 1986 im Rahmen der Bedarfslenkung männliche Absolventen anforderte, teilte das Kollegium Magdeburg dem Ministerium als besondere Wünsche mit: „weiblich, Mitglied der SED, zu Führungs- und Leitungsaufgaben geeignet. “ Der Anteil der weiblichen Mitglieder sollte bis 1990 um mindestens zwei erhöht werden, so der Plan. ${ }^{32}$

Seine Vorstellungen über die Anforderungen, die ein/e Bewerber/in grundsätzlich erfüllen musste, hat das Kollegium

17 Programm für die Auswertung der Erhebung unter den weiblichen Juristinnen in der Justiz, Bundesarchiv: DP-1/1542.

18 Bericht über den Einsatz und die Leistungen der Frauen im Bereich des Ministeriums der Justiz v. 20.8.1968, Bundesarchiv: DP-1/1653.

19 Bericht über den Einsatz und die Leistungen der Frauen im Bereich des Ministeriums der Justiz v. 20.8.1968, Bundesarchiv: DP-1/1653; vgl. auch: Düsing, Mechtild/Loroch, Stefanie, Die Lage der Anwältinnen nach 1945 in der Bundesrepublik und in der DDR, in: DAV (Hrsg.), Anwälte und ihre Geschichte, Tübingen 2011, S. 404 f.

20 Kaderprogramm des Kollegiums der Rechtsanwälte des Bezirkes Leipzig, Stand 1.10.1981, Bundesarchiv: DP-1/4469.

21 Vgl. hierzu auch: Düsing, Mechtild/Loroch, Stefanie, Die Lage der Anwältinnen nach 1945 in der Bundesrepublik und in der DDR, in: DAV (Hrsg.), Anwälte und ihre Geschichte, Tübingen 2011, S. 405.

22 Bericht des Kollegiums der Rechtsanwälte in Dresden im 1. Halbjahr 1964, Bundesarchiv: DP-1/1670.

23 Auskunft der Rechtsanwaltskammer Sachsen.

24 Entwurfsvorlage für die Kollegiumssitzung am 12.5.1965 v. 12.4.1965, Bundesarchiv: DP-1/4729. Laut der Entwurfsvorlage waren zum Stichtag 31.3.1965 insgesamt 447 Kollegienanwälte, 169 Einzelanwälte und 64 Rechtsbeistände tätig.

25 Einschätzung der Jahreshauptversammlungen 1965/66 der Kollegien der Rechtsanwälte, Protokoll v. 2.6.1966, Bundesarchiv: DP-1/1608.

26 Ebd., Bundesarchiv: DP-1/1608.

27 Ebd.

28 Einschätzung des Entwicklungsstandes des Kollegiums der Rechtsanwälte des Bezirkes Potsdam, 26.11.1976 Entwurf, Bundesarchiv: DP-1/3309.

29 Vermerk v. 10.2.1983 an die Hauptabteilung 7, Bundesarchiv: DP$1 / 4160$.

30 Kaderprogramm des Kollegiums der Rechtsanwälte des Bezirkes Leipzig, Stand 1.10.1981, Bundesarchiv: DP-1/4469.

31 Kaderperspektivplan des Kollegiums Schwerin bis 1990, Bundesarchiv: DP-1/4730.

32 Kollegium der Rechtsanwälte im Bezirk Magdeburg 2.12.1986 Beschluss Nr. 4/1987 zum Programm der Kader- und Zweigstellentwicklung 1986-1990, Bundesarchiv: DP-1/4730. 
Leipzig in seinem Kaderprogramm von 1981 festgehalten: der Nachweis aktiver gesellschaftlicher Tätigkeit, möglichst Parteizugehörigkeit, Abschluss des juristischen Staatsexamens mindestens mit der Note 2, Nachweis einer mehrjährigen erfolgreichen Tätigkeit als Jurist (Ausnahmen nur bei gelenkten Kadern) und möglichst nicht älter als 35 Jahre. ${ }^{33}$ Das „politisch-ideologische Niveau im Kollegium“ wurde besonders aufmerksam beobachtet. Im Kaderprogramm von 1981 ist festgehalten, dass „die Zusammensetzung der Mitglieder einen Bestand an Genossen von 59,18 Prozent aufweist.“ Dies veranlasste zu der Anmerkung: „Dadurch ist nicht nur der parteimäßige Einfluss gesichert, sondern auch die Möglichkeit, auf diese Mitglieder und über diese Mitglieder entsprechenden Einfluss zu gewinnen. “34 Das „richtige“ gesellschaftliche und politische Engagement der Kollegiumsmitglieder und der Aspiranten war also erwünscht, wenn nicht gar gefordert. Familiäre Bande im Hinblick auf die Übernahme eines Anwaltsbüros von einem Elternteil dagegen waren unerheblich, wie Unterlagen im Zusammenhang mit einem entsprechenden Antrag aus dem Jahr 1986 zeigen. Aufgrund gesundheitlicher Einschränkungen des Vaters sollte die Tochter diesen zunächst unterstützen und später die Zweigstelle übernehmen. Die Abteilung 7 des Ministeriums der Justiz vermerkte zu diesem Ansinnen: „Daß die Tochter die Zweigstelle des Vaters übernimmt, sollte eine Ausnahme bleiben.“ ${ }^{35}$

\section{Berufsalltag}

Ganz ohne familiäre Bindung, dafür aber fest entschlossen, begehrte die Juristin Renate Müller ${ }^{36}$ die Zulassung zum Kollegium in Magdeburg. Seit 1977 hatte sie sich mehrfach beim Vorstand um eine Zulassung beworben, stets erfolglos. Erst zum September 1982 wurde die damals 33-Jährige als Assistentin aufgenommen. In seiner Sitzung am 14. Juli 1983 sprach sich der Vorstand schließlich auch für die Zulassung als Anwältin aus und leitete den Antrag an das Ministerium der Justiz weiter. Diesem wurde von ministerieller Seite ebenfalls entsprochen. Und so wurde Renate Müller zum 1. September 1983 als erste Anwältin im Magdeburger Kollegium aufgenommen.

Als zunächst einzige Frau im Kollegium sei sie anfangs belächelt und durchaus kritisch beäugt worden, erzählt die - heute wieder - in Magdeburg tätige Rechtsanwältin. ${ }^{37}$ Damals bearbeitete sie hauptsächlich familienrechtliche, aber auch strafrechtliche Mandate. Der Arbeitsdruck sei hoch gewesen, die zeitliche Belastung ebenso. Erst nach etwa zwei, drei Jahren habe sie sich von ihren männlichen Kollegen akzeptiert gefühlt. Ihre hohen Umsätze dürften in diesem Zusammenhang nicht unerheblich gewesen sein, resümiert sie im Nachhinein. Und tatsächlich waren nach einer Einschätzung des Kollegiums Potsdam von 1976 die höchsten Gebühreneinnahmen in „Ehesachen“ zu erzielen. Strafrechtliche Mandate lagen dagegen noch deutlich hinter Wirtschafts- und Zivilstreitigkeiten. ${ }^{38}$

Anders als während ihrer ersten Jahre als Rechtsanwältin, in denen sie als Frau gegen einige Vorurteile zu kämpfen gehabt habe, so Liebold-Müller heute, habe sie sich während ihres Studiums nie als Fremdkörper gefühlt. Der Anteil weib- licher Studenten betrug, so erinnert sie sich, etwa ein Drittel. Mit neun Kommilitoninnen teilte sie sich die ersten Semester behelfsmäßig einen Klassenraum als Schlafstatt, da das Wohnheim in Halle-Neustadt noch nicht fertig gestellt war. Später waren sie dort zu dritt in einem Zimmer untergebracht.

Marxismus und Leninismus sowie eine Fremdsprache gehörten zu den Pflichtveranstaltungen im Grundstudium. Doch nicht jede Pflichtveranstaltung vermochte das studentische Interesse in gleicher Weise zu fesseln. Und so gelang es, die generelle Anwesenheitspflicht das ein oder andere Mal zu umgehen, indem man sich "gegenseitig vertrat", berichtet LieboldMüller über ihre Studienzeit.

Zum Zeitpunkt der Wende waren im Bezirk noch zwei weitere Kolleginnen tätig. Als die Mauer am 9. November 1989 fiel, war Liebold-Müller jedoch bereits ausgereist, erinnert sie sich. Ein Todesfall in der Familie sei für ihren Mann und sie die Gelegenheit gewesen, um im September die DDR zu verlassen. Erst Ende Dezember kehrte sie allein zurück, um den Verlust ihrer Anwaltszulassung zu verhindern. Da war ihre Wohnung noch versiegelt, erzählt sie rückblickend. Heute, fast drei Jahrzehnte später, ist Liebold-Müller noch immer in einer Kanzlei in Magdeburg tätig.

Das am 15. September 1990 in Kraft getretene Rechtsanwaltsgesetz $^{39}$ stellte die gesamte Tätigkeit der Anwaltschaft für das Gebiet der ehemaligen DDR auf eine neue gesetzliche Grundlage. Wie bereits nach dem zweiten Weltkrieg folgte auch nach dem Ende der DDR eine Phase der Überprüfung, die besondere Verstrickungen in das SED-Regime betraf. „Als Organ der Rechtspflege dürfen in der Rechtsanwaltschaft nur Personen tätig werden, deren Integrität außer Zweifel steht“, heißt es in einer Anfrage zur Zulassung als Rechtsanwalt im Landtag von Mecklenburg-Vorpommern aus dem Jahr 1991.40 Der Einigungsvertrag ordnete für die fünf neuen Bundesländer zunächst die Fortgeltung des Rechtsanwaltsgesetzes anstelle der Bundesrechtsanwaltsordnung an. Durch das Gesetz zur Anpassung der Rechtspflege im Beitrittsgebiet vom 26. Juni $1992^{41}$ waren schließlich die Weichen gestellt für ein einheitliches Rechtssystem im wieder vereinigten Deutschland.

\section{Schlussbetrachtungen}

Das tradierte Bild des Rechtsanwalts, unbewusst eigentlich immer männlich, ist aus den Köpfen noch immer nicht ganz

33 Kaderprogramm des Kollegiums der Rechtsanwälte des Bezirkes Leipzig, Stand 1.10.1981, Bundesarchiv: DP-1/4469.

34 Ebd., Bundesarchiv: DP-1/4469.

35 Vermerk des MdJ v. 2.7.1986, Bundesarchiv: DP-1/4470.

36 Liebold-Müller, Renate: geb. 7. Juli 1949 (geb. Müller), Lehre als Handelskauffrau, studierte von 1969 bis 1973 Staats- und Rechtswissenschaften an der Martin-Luther-Universität in Halle. Bis 1981 arbeitete sie als Justiziarin in einer Werkzeugmaschinenfabrik.

37 Die Darstellungen über die Tätigkeit und das Studium beruhen auf einem Interview der Autorin mit der Anwältin Renate Liebold-Müller am 21.10.2009 (unveröffentlicht).

38 Einschätzung des Entwicklungsstandes des Kollegiums der Rechtsanwälte des Bezirkes Potsdam, 26.11.1976 Entwurf, Bundesarchiv: DP-1/3309.

39 GBI. der DDR 1990, Teil I Nr. 61, S. 1504 ff.

40 Drucks. 1/1115 v. 18.12.1991 im Landtag von Mecklenburg-Vorpommern.

41 BGBI. I 1992, Nr. 29, S. 1147. 
ausradiert; doch es verblasst zusehends und gewinnt gleichzeitig neue Konturen, weiblichere Konturen: „Das eherne Leitbild eines Zigarre schmaukenden Kanzlei-Lenkers, der im Clubsessel einen schweren Cognac-Schwenker kreisen lässt, hat deutliche Risse bekommen." - lautet auch das Fazit von Dommer in einem Artikel mit dem Titel „Kanzleien, Kinder und Karrieren“. ${ }^{42} \mathrm{Zu}$ Beginn des 21. Jahrhunderts ist „Frau Rechtsanwältin“ also nicht mehr nur Angestellte, sondern immer häufiger selbst Chefin.

Die Zahl der Frauen, die sich für den Anwaltsberuf entscheiden, steigt seit Jahrzehnten trotz anhaltend schwieriger Arbeitsmarktlage. Diese Entwicklung war sowohl im Westteil Deutschlands als auch in der DDR zu beobachten und setzt sich noch immer kontinuierlich fort im wieder geeinten Deutschland.

Vorhersehbar war dies zu Beginn des 20. Jahrhunderts nicht, für einige nicht einmal vorstellbar: „Die Frau eignet sich nicht zur Rechtsanwaltschaft oder zum Richteramt. Ihre Zulassung würde daher zu einer Schädigung der Rechtspflege führen und ist aus diesem Grund abzulehnen. "43 - Die Vertreterversammlung der Anwaltschaft offenbarte im Jahr 1922 eine äußerst rigide, wenn auch nicht einstimmige Haltung zur Aufnahme von Frauen. ${ }^{44}$ Der viel zitierte Beschluss wurde mit 45 zu 22 Stimmen verabschiedet. Er konnte jedoch nicht aufhalten, was längst in Gang gesetzt war: Im Dezember 1922 wurde Maria Otto als erste Frau in Deutschland zur Rechtsanwaltschaft zugelassen, ${ }^{45}$ ihr sollten bis heute viele Tausende folgen.

Dies wurde möglich, weil sich die politischen Strukturen in Ost- und Westdeutschland veränderten, gesellschaftliche Denkmuster, angestoßen durch individuelles Vordenkertum, aufbrachen und standesrechtliche Notwendigkeiten erkannt wurden. Es ist den Anwältinnen gelungen, sich mehr und mehr in der Anwaltschaft zu etablieren. In den Hörsälen der Universitäten ist eine männliche Übermacht nicht mehr feststellbar. In juristischen Führungspositionen, auch im Hinblick auf die partnerschaftliche Beteiligung in Kanzleien und ein geschlechtsunabhängiges Einkommen, ist dieses Ziel indes noch nicht erreicht. Die an dem abrissartig aufgezeigten Entwicklungsprozess Beteiligten haben eines jedoch eindrucksvoll unter Beweis gestellt: Eine Schädigung der Rechtspflege ist aufgrund der Zulassung der Frauen zur Rechtsanwaltschaft nicht eingetreten, im Gegenteil!
Die Süddeutsche Zeitung zitiert in einem Artikel aus dem Jahr 2011 mit dem Titel „Karriere als Anwältin - bitte ohne Kinder" den Partner einer Wirtschaftskanzlei mit den Worten: „Wir brauchen die Frauen unbedingt, also müssen wir uns der gesellschaftlichen Verantwortung stellen und mit den alten Mustern brechen. “46

Die ehedem geäußerten Vorbehalte männlicher Kollegen waren demnach lediglich Ausdruck einer wenig rühmlichen, noch weniger weitsichtigen, geradezu obstinaten Haltung, die sich als dringend korrekturbedürftig erwies. ${ }^{47}$ Doch der „Geschlechterkampf im Gerichtssaal“" wie Patrick Ruppert es in seinem Artikel „Jungs gegen Mädchen“48 bezeichnet, wird auch in Zukunft weitergehen, Frau und Mann bleiben nun einmal Frau und Mann. Und das Thema ist präsent wie nie: 2008 wurde die Ausstellung "Justitia ist eine Frau“ in Leipzig eröffnet. Die AdVoice, die Zeitschrift des Forums Junge Anwaltschaft im Deutschen Anwaltsverein, titelte in einer Ausgabe aus dem Jahr 2009 „Frauen sind die besseren Männer - Über den Unterschied zwischen Rechtsanwälten und Rechtsanwältinnen "49 und immer mehr Kanzleien machen sich Gedanken über Teilzeitlösungen für Associates wie auch für Partner. ${ }^{50}$ Angesichts dessen bleibt am Ende nur noch eines zu konstatieren: Und sie eignet sich doch!

42 Dommer, Martin, Kanzleien, Kinder und Karrieren, in: Anwaltsblatt Karriere, WS og/10, S. 59-60.

43 Protokoll der 14. Vertreterversammlung des DAV, in: JW 1922, 1241 (1247 f.).

44 Vgl. auch: Düsing, Mechtild/Loroch, Stefanie, Die Lage der Anwältinnen nach 1945 in der Bundesrepublik und in der DDR, in: DAV (Hrsg.), Anwälte und ihre Geschichte, Tübingen 2011, S. 396.

45 Heinicke, Petra, Dr. Maria Otto, die erste deutsche Rechtsanwältin, in: BRAK-Mitteilungen, 6/2008, X-XI.

46 Ustorf, Anne-Ev, Karriere als Anwältin - bitte ohne Kinder, in: sueddeutsche.de, Artikel v. 12.6.2011, erhältlich im Internet: http://www. sueddeutsche.de/karriere/2.220/frauen-als-partner-in-kanzleienkarriere-als-anwaeltin-bitte-ohne-kinder-1.1107116 (Zugriff: 27.12.2011).

47 Düsing, Mechtild/Loroch, Stefanie, Die Lage der Anwältinnen nach 1945 in der Bundesrepublik und in der DDR, in: DAV (Hrsg.), Anwälte und ihre Geschichte, Tübingen 2011, S. 398.

48 Ruppert, Patrick, Jungs gegen Mädchen, in: AdVoice, o4/og, S. 10-11.

49 Ders., Frauen sind die besseren Männer, in: AdVoice, 04/o9, S. 4-5.

50 Dommer, Martin: Kanzleien, Kinder und Karrieren, in: Anwaltsblatt Karriere, WS o9/10, S. 59-6o; Ustorf, Anne-Ev: Karriere als Anwältin - bitte ohne Kinder, ebd.

\section{Quantität als Erfolgsgeschichte? Frauen in der Justiz der DDR}

\author{
Dr. Marion Röwekamp \\ Feodor Lynen Fellow der Alexander von Humboldt Stiftung am \\ Colmex, CIESAS und der UNAM, Mexico City
}

Die Geschichte der Frauen in der Justiz in der DDR stand im Erbe der Geschichte der deutschen Juristinnen, deren Berufs- einstieg in die Justiz vor 1945 von einer besonderen Härte gekennzeichnet gewesen war. Neben dem Beruf des Hochschulprofessors gehörten die Berufe in der Justiz zu den besonders gehüteten Männerdomänen. ${ }^{1}$ Die in der Welt einmalig hohen Zahlen von Richterinnen und Staatsanwältinnen in der DDR sind insbesondere vor diesem Hintergrund eine weibliche Er- 